\title{
General Control of Arginine Biosynthetic Enzymes in Neurospora crassa
}

\author{
By HARRY J. FLINT*† AND BARBARA F. KEMP \\ Department of Genetics, University of Nottingham, University Park, Nottingham NG7 2RD
}

(Received 31 July 1980)

\begin{abstract}
The response of six amino acid synthetic enzymes, including four concerned with arginine synthesis, one with histidine synthesis and one with lysine synthesis, to conditions of histidine and arginine limitation and to exogenously provided amino acids is described in Neurospora crassa. The activities of all these enzymes increased in response to lowered levels of histidine or arginine, but showed little or no repression in wild-type cultures supplemented with casein hydrolysate. The activity of glucose-6-phosphate dehydrogenase (an enzyme not concerned with amino acid synthesis) remained unaffected by any of these conditions. In the case of the four arginine synthetic enzymes evidence is presented that suggests the 'general' system implicated in the 'cross-pathway' response to histidine is also entirely responsible for the derepression occurring under conditions of arginine limitation. Further investigations into the control of one enzyme, ornithine carbamoyltransferase, are also reported. These show that the enzyme is stable, and suggest that its derepression in response to histidine limitation entails new protein synthesis and involves control at a stage prior to translation.
\end{abstract}

\section{INTRODUCTION}

The regulation of arginine biosynthesis in Neurospora crassa has been the subject of extensive investigations (Cybis \& Davis, 1975; Davis et al., 1978; Flint et al., 1980) but the mechanisms that control the levels of enzymes in this pathway have remained obscure. The activities of the eight enzymes involved have been shown to increase progressively with decreasing mycelial arginine content in arginine bradytrophic strains (Barthelmess et al., 1974; Cybis \& Davis, 1975). In addition, there is evidence to show that the activities of at least two arginine synthetic enzymes become elevated, together with many enzymes involved in histidine and tryptophan synthesis, under conditions of histidine or tryptophan limitation (Carsiotis \& Jones, 1974; Carsiotis et al., 1974). Such 'cross-pathway' regulatory phenomena have also been observed in Saccharomyces cerevisiae (Schürch et al., 1974; Delforge et al., 1975) and in Aspergillus nidulans (Piotrowska, 1980) and have been taken to imply the existence of a 'general' regulatory system whereby the enzymes of many different amino acid pathways respond to the limitation of any one of a number of amino acids. This contrasts with the 'pathway-specific' control that predominates in bacteria, whereby the enzymes of a particular pathway respond only to the end-product of that pathway.

This paper reports the response of a number of amino acid synthetic enzymes, including four of the enzymes involved in arginine synthesis, to conditions of histidine and arginine limitation, and to the presence of exogenously provided amino acids. This allows an assessment of the role of general control in governing enzyme levels in the arginine pathway. Further studies on one of the enzymes (ornithine carbamoyltransferase) are also described that examine enzyme stability, the requirement of de novo synthesis for derepression and the

\footnotetext{
† Present address: Department of Biology, Cavehill Campus, University of the West Indies, Barbados, West Indies.

$\ddagger$ Present address: Department of Genetics, University of Leeds, Leeds LS2 9JT.
} 
level at which general control of enzyme synthesis is exerted. These are based on the change in enzyme activity observed in derepressed cultures following the inhibition of protein synthesis or the restoration of repressive conditions.

\section{METHODS}

Strains. The St. Lawrence wild-type strain of $N$. crassa and the 'leaky' arginine double mutant arg- $1 \mathrm{r} 3 \mathrm{arg}-10 \mathrm{rl}$ were obtained from $\mathrm{H}$. Kacser. The origin and properties of the latter strain were described by Barthelmess et al. (1974). The arginine auxotroph $\mathrm{arg}-5$ and the histidine auxotroph his-2 were obtained from the Fungal Genetics Stock Center, Humboldt State University Foundation, Arcata, California. U.S.A.

Growth conditions and harvesting. Mycelium was grown in 11 conical flasks under constant agitation (at 200 rev. $\min ^{-1}$ ) in a Gallenkamp orbital shaker at $29{ }^{\circ} \mathrm{C}$. Each flask contained $200 \mathrm{ml}$ Vogel's minimal medium and $4.5 \mathrm{~g}$ glucose as a carbon source, and was inoculated with about $10^{7}$ conidia from a suspension freed of mycelial debris by passage through a glass wool filter. Mycelium was harvested at least $16 \mathrm{~h}$ after inoculation by filtering the contents of complete flasks under suction through three layers of filter paper in a Buchner funnel. The mycelial pads were removed, rapidly frozen to $-30^{\circ} \mathrm{C}$ and then lyophilized. In this form mycelium could be stored under desiccation at $-30^{\circ} \mathrm{C}$ for several weeks without loss of enzyme activity. The number of harvests taken for assay at each time in the different experiments is given in Results.

Enzyme assays. All enzyme assays were performed on dialysed extracts prepared from lyophilized mycelium. Powdered mycelium $(10 \mathrm{or} 20 \mathrm{mg}$ ) was homogenized in $1 \mathrm{ml} 0.05 \mathrm{M}$-Tris/ $\mathrm{HCl}$ buffer $(\mathrm{pH} 7.5)$ containing $0.1 \mathrm{~mm}$-EDTA and $0.5 \mathrm{~mm}$-dithiothreitol, and the homogenate was centrifuged at $15 \mathrm{~min}$ at $20000 \mathrm{~g}$ in a refrigerated centrifuge. The supernatant was then dialysed overnight against $0.01 \mathrm{M}$-Tris/ $\mathrm{HCl}$ buffer $(\mathrm{pH} 7.5)$ at $4{ }^{\circ} \mathrm{C}$. Assays were carried out immediately after dialysis using appropriate dilutions of the extract and were done at $37^{\circ} \mathrm{C}$ unless stated otherwise.

Ornithine carbamoyltransferase (EC 2.1.3.3) was assayed by the method of Davis (1962) but using the procedure of Marsh et al. (1965) to measure the citrulline formed. Acetylornithine aminotransferase (EC 2.6.1.11) was assayed by the method of Vogel \& Jones (1970).

Glutamate acetyltransferase (EC 2.3.1.35) was assayed by measuring the formation of acetyl| ${ }^{14} \mathrm{C}$ lglutamate from $\mid{ }^{14} \mathrm{C}$ |glutamate as described by Denes (1970). Argininosuccinase (argininosuccinate lyase: EC 4.3.2.1) activity was determined by the formation of $\mid{ }^{14} \mathrm{C}$ largininosuccinate from $\left[{ }^{14} \mathrm{C}\right]$ arginine. The assay mixture (final volume $0.3 \mathrm{ml}$ ) contained $20 \mathrm{mM}$-sodium fumarate, $20 \mathrm{mM}$-L-Iguanido ${ }^{14} \mathrm{C}$ |arginine (specific activity $8 \times 10^{4}$ c.p.m. $\mu \mathrm{mol}^{-1}$ ), $0.033 \mathrm{M}$-phosphate buffer ( $\mathrm{pH} \mathrm{7.4)}$ and sufficient dialysed extract to give a protein concentration of about $1.2 \mathrm{mg} \mathrm{m}^{-1}$. The reaction was initiated by the addition of extract, and stopped after 20 min incubation by the addition of $0.05 \mathrm{ml} 30 \%(\mathrm{w} / \mathrm{v})$ sulphosalicylic acid. The resulting mixture was centrifuged ( $1000 \mathrm{~g}$ for $10 \mathrm{~min}$ ) and the supernatant was passed through a Dowex 50- $\mathrm{X8}\left(\mathrm{H}^{+}\right)$column (a Pasteur pipette plugged with glass wool and half filled with resin) that had been pre-equilibrated at $\mathrm{pH} 5.0$ with sodium citrate buffer $\left(0.2 \mathrm{M}-\mathrm{Na}^{+}\right)$. The same buffer was used for elution. Arginine was retained, while argininosuccinate was eluted and collected in the first $4 \mathrm{ml}$ of eluant to pass through the column after discarding the sample volume. Tubes that received either no fumarate, no extract or boiled extract were used as controls. The production of $\left[{ }^{14} \mathrm{C}\right.$ largininosuccinate was linear with time of incubation and protein concentration under the conditions used. It proved necessary to purify both the $\mid{ }^{14} \mathrm{C}$ |arginine used for this assay and the $\left|{ }^{14} \mathrm{C}\right|$ glutamate used in the acetyltransferase assay prior to use by passage through Dowex columns. Radiochemicals were obtained from The Radiochemical Centre, Amersham.

Saccharopine dehydrogenase $\left(\mathrm{NAD}^{+}\right.$, lysine-forming; EC 1.5.1.7) was assayed at $28^{\circ} \mathrm{C}$ by the method of Broquist (1971) and glucose-6-phosphate dehydrogenase (EC 1.1.1.49) was assayed at $25^{\circ} \mathrm{C}$ by the method of Scott (1975). Histidinol dehydrogenase (EC 1.1.1.23) was assayed using the tetrazolium dye method of Martin (1971).

A unit of enzyme activity represents the formation of $1 \mu \mathrm{mol}$ product $\min ^{-1}$ in the case of ornithine carbamoyltransferase, argininosuccinase, glutamate acetyltransferase and acetylornithine aminotransferase. For the remaining enzymes a unit is equivalent to a rate of change of 0.01 absorbance units $\min ^{-1}$. Protein was determined by the Lowry method, using bovine serum albumen for calibration.

Amino acid pools. Amino acid analysis was performed using an autoanalyser on the supernatants obtained after homogenizing lyophilized mycelium in $5 \%(\mathrm{w} / \mathrm{v})$ sulphosalicylic acid, as described previously (Flint et al., 1980). In some cases the method of Van Pilsum et al. (1965) was used to determine mycelial arginine. For this purpose, $10 \mathrm{mg}$ of powdered, lyophilized mycelium was homogenized in $1.2 \mathrm{ml} 5 \%(\mathrm{w} / \mathrm{v})$ trichloroacetic acid, kept on ice for $30 \mathrm{~min}$, and then centrifuged at $1000 \mathrm{~g}$ for $15 \mathrm{~min}$. A 5 - or 10 -fold dilution of the supernatant was then used for the determination of arginine. employing standard solutions containing the appropriate concentrations of trichloroacetic acid. Estimates obtained by this method were entirely consistent with those obtained using the autoanalyser. 
Table 1. Amino acid pools in cultures treated with 3-amino-1,2,4-triazole 3-Amino-1,2,4-triazole ( $3 \mathrm{AT}$; final concentration $2.5 \mathrm{~mm}$ ) was added to wild-type cultures after $17 \mathrm{~h}$
growth on minimal medium. The values represent the means of two replicate harvests.

\begin{tabular}{|c|c|c|c|}
\hline \multirow[b]{2}{*}{ Amino acid } & \multicolumn{3}{|c|}{ Pool size $\left[\mu \mathrm{mol}(\mathrm{g} \text { dry } w \mathrm{t})^{-1}\right]$} \\
\hline & $\begin{array}{l}\text { Minimal } \\
\text { medium }\end{array}$ & $\begin{array}{c}1 \mathrm{~h} \text { after } \\
\text { 3AT addition }\end{array}$ & $\begin{array}{c}6 \mathrm{~h} \text { after } \\
\text { 3AT addition }\end{array}$ \\
\hline Histidine & $4 \cdot 2$ & $1 \cdot 2$ & $1 \cdot 1$ \\
\hline Arginine & 31 & 66 & 84 \\
\hline Ornithine & 29 & 25 & 37 \\
\hline Citrulline & $5 \cdot 2$ & $3 \cdot 2$ & $2 \cdot 1$ \\
\hline Lysine & $5 \cdot 7$ & 9.9 & $30 \cdot 1$ \\
\hline Leucine & $1 \cdot 1$ & 1.9 & $3 \cdot 6$ \\
\hline Valine & $6 \cdot 4$ & $8 \cdot 3$ & $17 \cdot 1$ \\
\hline Isoleucine & $1 \cdot 1$ & $1 \cdot 2$ & $1 \cdot 7$ \\
\hline Glutamate & 131 & 109 & 149 \\
\hline Glutamine & 56 & 84 & 87 \\
\hline Alanine & 82 & 95 & 124 \\
\hline Tyrosine & $1 \cdot 3$ & ND & $1 \cdot 4$ \\
\hline Phenylalanine & 0.5 & ND & 1.0 \\
\hline Tryptophan & $0 \cdot 3$ & ND & 0.2 \\
\hline
\end{tabular}

\section{RESULTS}

\section{Histidine-mediated control}

The control of arginine synthetic enzymes through histidine-mediated cross-pathway regulation was investigated in wild-type cultures treated with 3-amino-1,2,4-triazole (3AT). $3 \mathrm{AT}$ is known to inhibit the histidine synthetic enzyme imidazoleglycerol-phosphate dehydratase; this in turn causes reductions in mycelial histidine content and in the state of charging of histidyl tRNA (Spurgeon \& Matchett, 1977). Table 1 shows the effect of 3AT on amino acid pools in wild-type cultures. Histidine decreased fourfold; the arginine precursors ornithine and citrulline showed, respectively, no change and a twofold decrease. The other amino acids examined either increased or remained constant. Arginine, lysine, leucine and valine had all increased some threefold or more after $6 \mathrm{~h}$ exposure.

Derepression of the arginine synthetic enzymes ornithine carbamoyltransferase and argininosuccinase followed the addition of $3 \mathrm{AT}$ to cultures growing on minimal medium (Fig. $1 a, b)$. ('Derepression' and 'repression' are used in this paper in the operational sense, to refer to an increase in enzyme activity consequent upon decreasing an amino acid level and a decrease in enzyme activity consequent upon raising an amino acid level, without implying a particular mechanism.) In addition, the presence of 3AT inhibited growth (Fig. 1c). The addition of histidine [which rapidly restores normal mycelial histidine levels (see Fig. 4)] $1 \mathrm{~h}$ before 3AT addition abolished derepression and almost completely eliminated the effect of $3 \mathrm{AT}$ on growth. The addition of arginine at the same stage failed to abolish the derepression and only slightly alleviated the effect of $3 \mathrm{AT}$ on growth. The activities of five other enzymes, determined in the same experiment for harvests taken 6 and $23 \mathrm{~h}$ after $3 \mathrm{AT}$ addition, are shown in Table 2 . In addition to the two arginine synthetic enzymes discussed already, glutamate acetyltransferase and acetylornithine aminotransferase also showed histidinemediated derepression. Saccharopine dehydrogenase (involved in lysine synthesis) and histidinol dehydrogenase (involved in histidine synthesis) also responded to 3AT. Glucose6-phosphate dehydrogenase, on the other hand, did not, confirming that histidine limitation does not cause indiscriminate changes in all enzyme activities.

The increase noted in the mycelial arginine pool, and in certain other amino acids, following 3AT addition (Table 1) could be due to increased endogenous synthesis resulting 


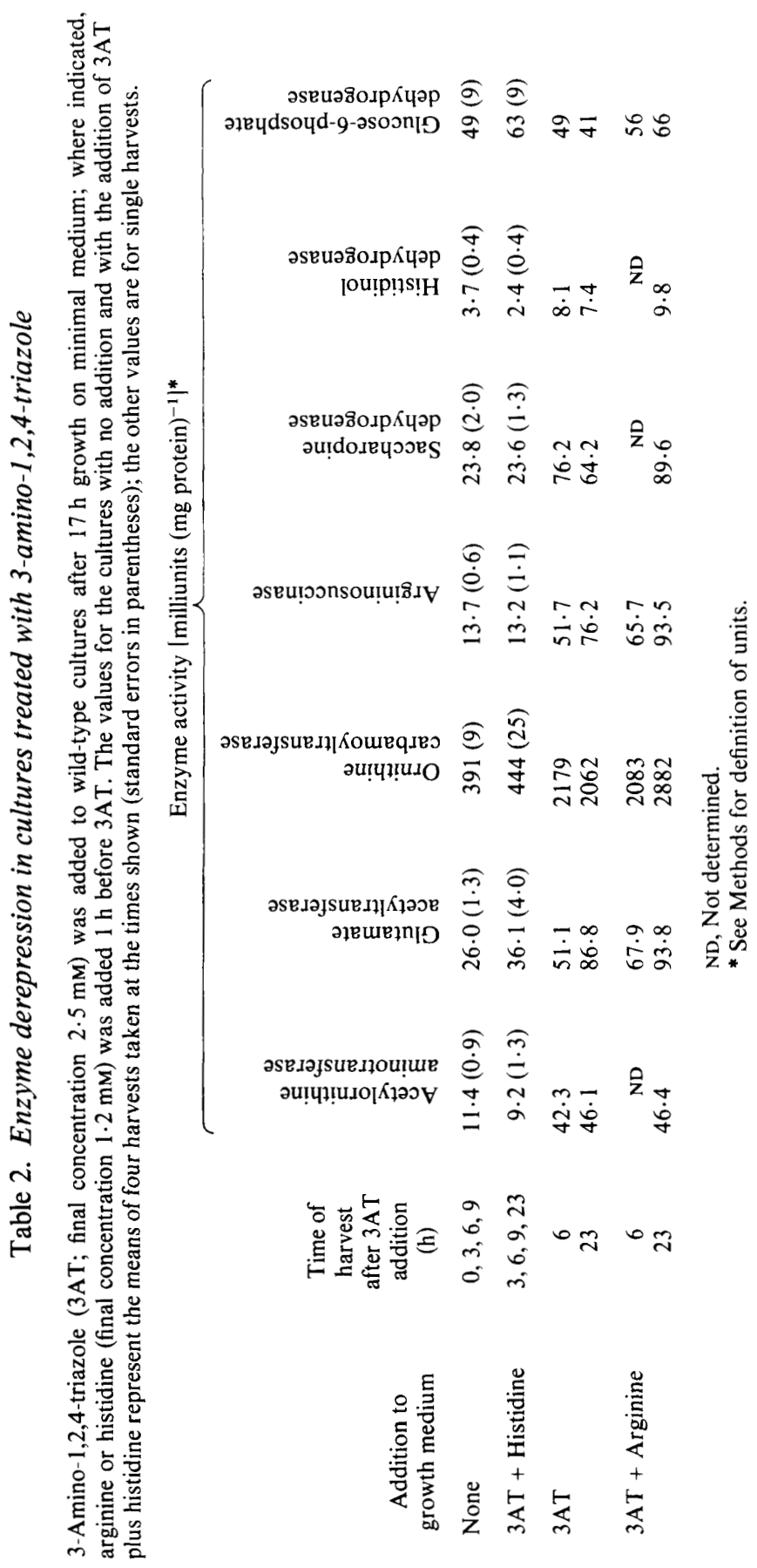



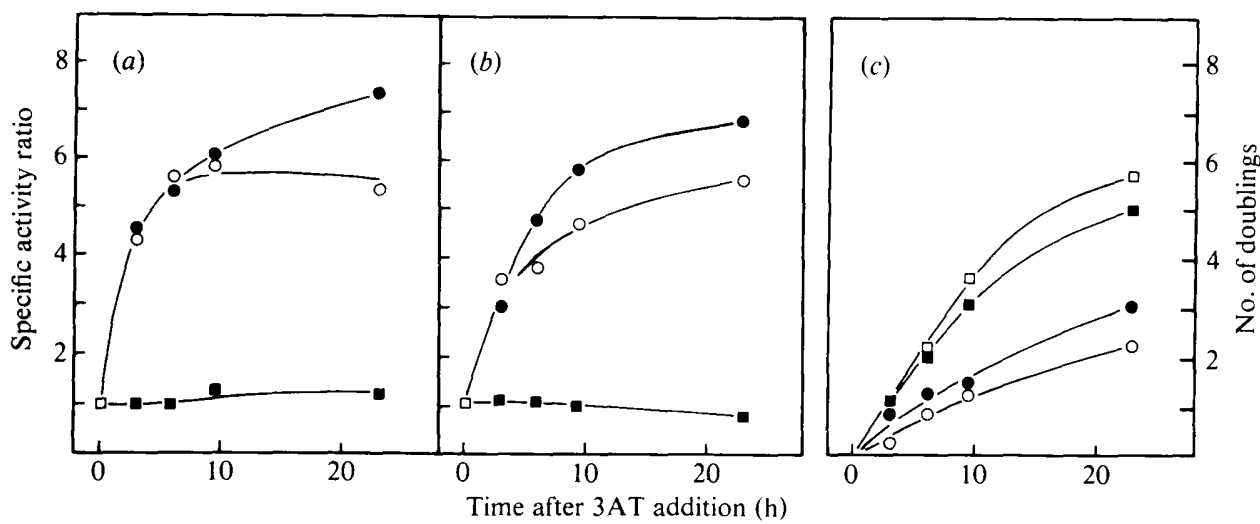

Fig. 1. Enzyme derepression and growth in wild-type cultures treated with 3-amino-1,2,4-triazole (3AT). Specific activity ratios (enzyme activities expressed relative to those for minimal-grown cultures) of ornithine carbamoyltransferase $(a)$ and argininosuccinase $(b)$, and growth $(c)$ are shown for cultures treated with $3 \mathrm{AT}$ (final concentration $2.5 \mathrm{~mm})(\mathrm{O}), 3 \mathrm{AT}(2.5 \mathrm{mM})$ plus arginine $(1.2 \mathrm{~mm})(\mathrm{O})$ or $3 \mathrm{AT}$ $(2.5 \mathrm{~mm})$ plus histidine $(1.2 \mathrm{mM})(\square)$. $3 \mathrm{AT}$ was added after $17 \mathrm{~h}$ growth on minimal medium; arginine or histidine was added $1 \mathrm{~h}$ before $3 \mathrm{AT}$. The values for minimal-grown cultures $(\square)$ represent the mean of four harvests, except in $(c)$ where they are for single harvests; the values for treated cultures are for single harvests. The numbers of doublings in $(c)$ were derived from $\log _{2}\left(P_{t} / P_{0}\right)$ where $P_{0}$ is the total culture protein at the time of $3 \mathrm{AT}$ addition and $P_{\mathrm{t}}$ is the total culture protein at a subsequent time $t$.

from enzyme derepression. However, it could also be attributable to the reduced utilization of amino acids for protein synthesis that must accompany the inhibition of growth by $3 \mathrm{AT}$. It is not possible to decide between these two explanations on the evidence available.

\section{Enzyme levels under repressive conditions}

The previous section examined the derepression of amino acid synthetic enzymes in response to a decrease in the mycelial histidine pool. In order to determine whether an increase in mycelial histidine, or in any other amino acid, caused repression, enzyme activities were determined for cultures supplemented with casein hydrolysate (Table 3 ). The activities of all the enzymes during growth with casein hydrolysate were close to their fully repressed levels during growth on minimal medium, although a slight, but statistically significant $(P<0.01)$, repression (of about $40 \%$ ) was observed for glutamate acetyltransferase and acetylornithine aminotransferase. Thus, increases in amino acid pools to levels above those found for minimal-grown cultures (as occur in histidine-limited cultures - see Table 1) are expected to have only a small effect on enzyme levels.

\section{Arginine-mediated control}

In the absence of a suitable inhibitor of arginine synthesis, enzyme derepression in response to arginine limitation was examined in an arginine auxotrophic ( $\arg -5)$ strain grown on low-arginine medium (Table 4). Arginine limitation resulted in the cross-pathway derepression of histidinol dehydrogenase and saccharopine dehydrogenase, in addition to causing the derepression of arginine synthetic enzymes. On high-arginine medium ( $2 \mathrm{~mm})$ the $\arg -5$ strain showed activities close to those observed for the wild-type strain grown on minimal medium, although the mycelial arginine level was about fourfold higher in the auxotroph. Previous studies have shown that growth of the wild-type strain on medium containing arginine does not cause the four arginine enzymes to become repressed below their minimal growth levels (Cybis \& Davis, 1975; Barthelmess et al., 1974).

The specific activity ratios (i.e. the activities expressed relative to those for minimal-grown wild-type cultures) of argininosuccinase, saccharopine dehydrogenase and histidinol dehydrogenase in mycelium harvested at various times after the onset of histidine- or 

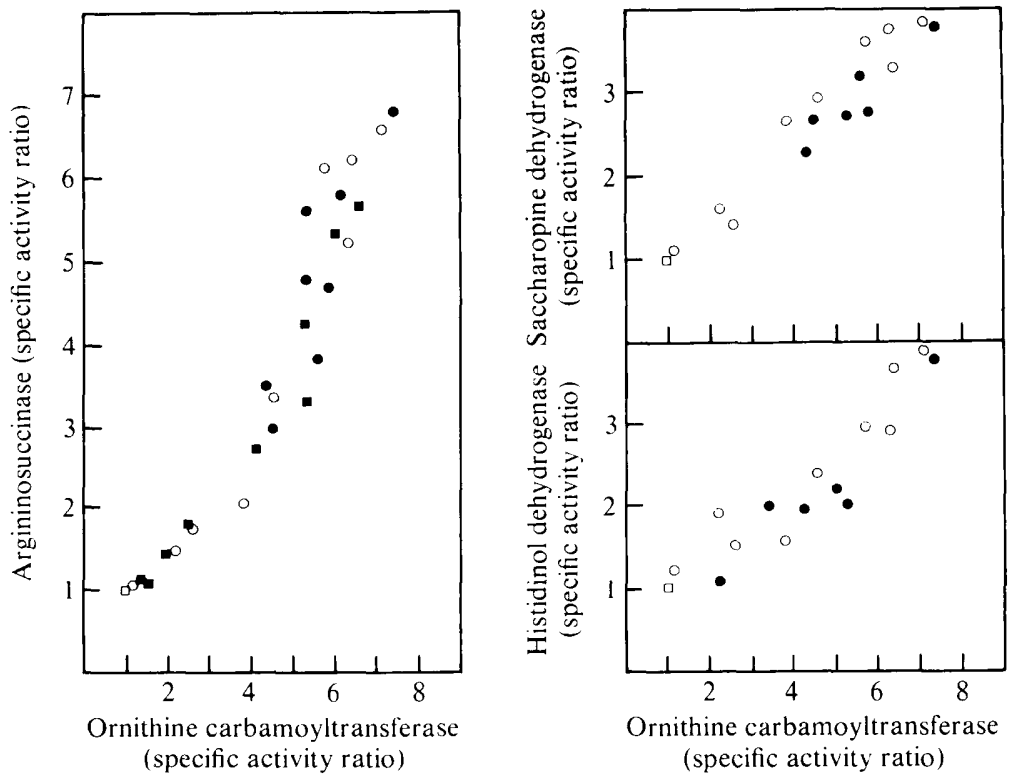

Fig. 2. Relationships between the activity of ornithine carbamoyltransferase and the activities of three other amino acid synthetic enzymes under conditions of histidine and arginine limitation. Specific activity ratios are shown, taking the values for minimal-grown wild-type cultures (see Table 2) as 1.0 ( $\square$ ). Each point represents a single harvest for the wild-type grown in the presence of $3 \mathrm{AT}(\mathrm{O})$ or for arg-5 grown on low levels $(0.12$ or $0.04 \mathrm{~mm})$ of arginine (O). Data obtained from the growth of a histidine auxotroph (his-2) on low levels $(0.06$ or $0.03 \mathrm{~mm})$ of histidine $(\boldsymbol{a})$ are also included for the argininosuccinase/ornithine carbamoyltransferase plot.

arginine-mediated derepression are plotted against ornithine carbamoyltransferase activity in Fig. 2. In all three cases the relationships for arginine- and histidine-mediated derepression are similar. This is consistent with the derepression of these four enzymes being governed by a single common system of control, irrespective of which amino acid is responsible.

The data for glutamate acetyltransferase are less extensive, but the specific activity ratios observed for this enzyme were between $50 \%$ and $66 \%$ of those found for ornithine carbamoyltransferase both for the $\arg$ - 5 strain grown on low-arginine medium and after prolonged exposure of the wild-type strain to 3AT (Table 4). Acetylornithine aminotransferase could not be determined in the $\arg -5$ strain, as $\arg -5$ is its structural locus. The specific activity ratios for this enzyme in arginine bradytrophic strains are about $30 \%$ higher than those shown by glutamate acetyltransferase. Thus there is no evidence to suggest that the derepression of these two enzymes, relative to that of the other amino acid synthetic enzymes studied here, differs under conditions of histidine and arginine limitation.

\section{Stability of ornithine carbamoyltransferase}

Ornithine carbamoyltransferase (OCTase) was chosen for further study because of its ease of assay and high activity in mycelial extracts. In order to determine whether this enzyme was subject to degradation in growing cultures the decline in derepressed OCTase activity was followed in an arginine bradytrophic strain after the addition of arginine to the medium (Fig. 3 ). The strain used carried two mutant genes (arg-1r3, arg-10rl) that result in severely reduced activities for the arginine pathway enzymes argininosuccinate synthetase and argininosuccinase respectively (Barthelmess et al., 1974). As a result this strain displays a decreased arginine pool and elevated activities for many amino acid biosynthetic enzymes, 


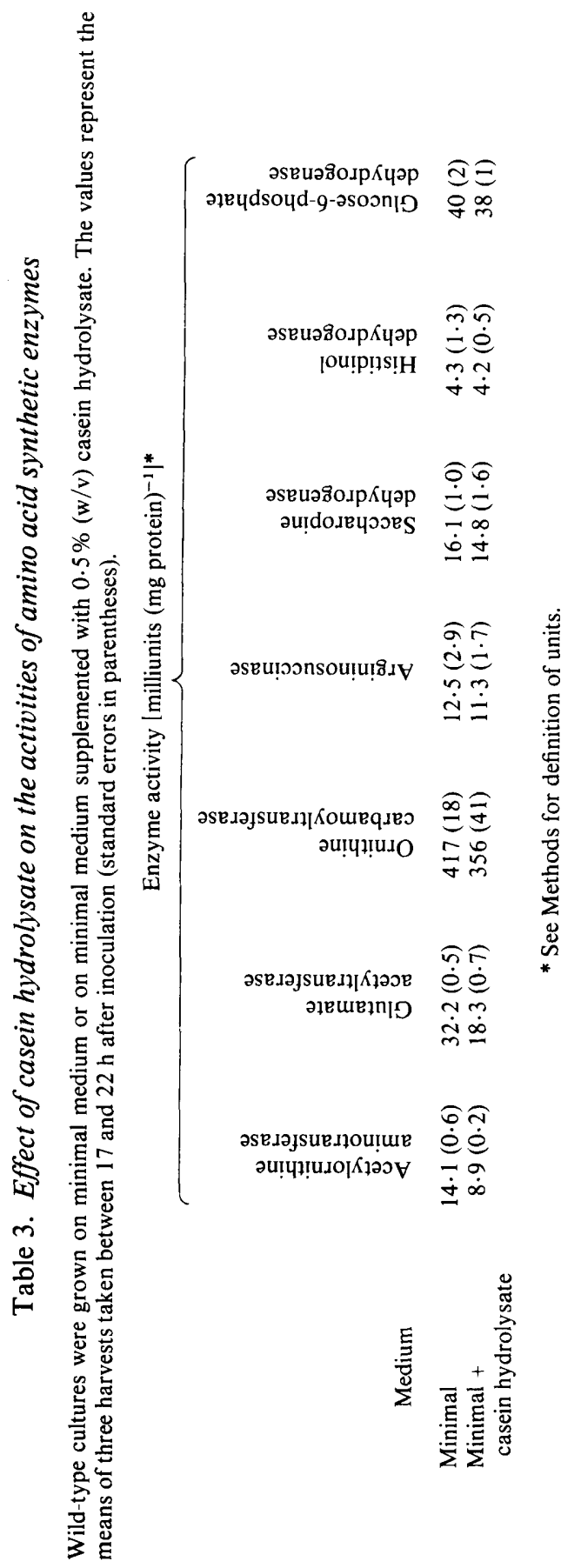


Table 4. Enzyme derepression in response to arginine limitation in an arginine auxotroph (arg-5)

Cultures of the $\arg -5$ strain grown on high-arginine medium ( $2 \mathrm{~mm}$ ) were harvested at times up to $28 \mathrm{~h}$ after inoculation, while still in the exponential phase of growth. Those grown on low-arginine medium $(0.12$ or $0.04 \mathrm{~mm})$ were harvested after $40 \mathrm{~h}$, when growth had virtually ceased due to exhaustion of the arginine provided. Each value is for a single harvest except for the $2 \mathrm{mM}$-arginine incubations (means of four harvests). Data for 3AT-treated wild-type cultures (based on the data shown in Table 2 for harvests taken $23 \mathrm{~h}$ after $3 \mathrm{AT}$ addition) are shown for comparison. The specific activity ratios show the enzyme activities relative to those for minimal-grown wild-type cultures (see Table 2).

\begin{tabular}{|c|c|c|c|c|c|c|c|c|}
\hline & & & & $\mathrm{Sp}$ & activ & atio & & \\
\hline Strain & $\begin{array}{l}\text { Initial } \\
\text { arginine } \\
\text { concn in } \\
\text { growth } \\
\text { medium } \\
\text { (mM) }\end{array}$ & 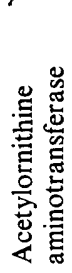 & 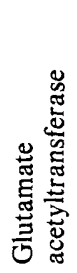 & 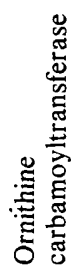 & 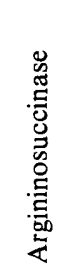 & 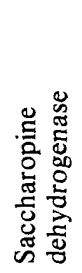 & 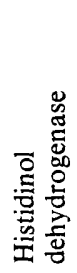 & 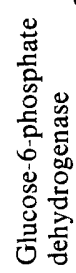 \\
\hline $\arg -5$ & $\begin{array}{l}2 \\
0 \cdot 12 \\
0.04\end{array}$ & - & $\begin{array}{l}1 \cdot 18 \\
3 \cdot 8 \\
4 \cdot 7\end{array}$ & $\begin{array}{l}1 \cdot 13 \\
6 \cdot 4 \\
7 \cdot 1\end{array}$ & $\begin{array}{l}1 \cdot 02 \\
6 \cdot 2 \\
6 \cdot 6\end{array}$ & $\begin{array}{l}1 \cdot 18 \\
3 \cdot 3 \\
3 \cdot 8\end{array}$ & $\begin{array}{l}1.22 \\
3.7 \\
3.9\end{array}$ & $\begin{array}{l}0.98 \\
0.82 \\
0.90\end{array}$ \\
\hline $\begin{array}{l}\text { 3AT-treated } \\
\text { wild-type }\end{array}$ & $\begin{array}{l}0 \\
1 \cdot 2\end{array}$ & $\begin{array}{l}4 \cdot 0 \\
4 \cdot 1\end{array}$ & $\begin{array}{l}3 \cdot 3 \\
3 \cdot 6\end{array}$ & $\begin{array}{l}5 \cdot 3 \\
7 \cdot 4\end{array}$ & $\begin{array}{l}5 \cdot 6 \\
6 \cdot 8\end{array}$ & $\begin{array}{l}2.7 \\
3.8\end{array}$ & $\begin{array}{l}2 \cdot 0 \\
3 \cdot 8\end{array}$ & $\begin{array}{l}0.84 \\
1 \cdot 35\end{array}$ \\
\hline
\end{tabular}

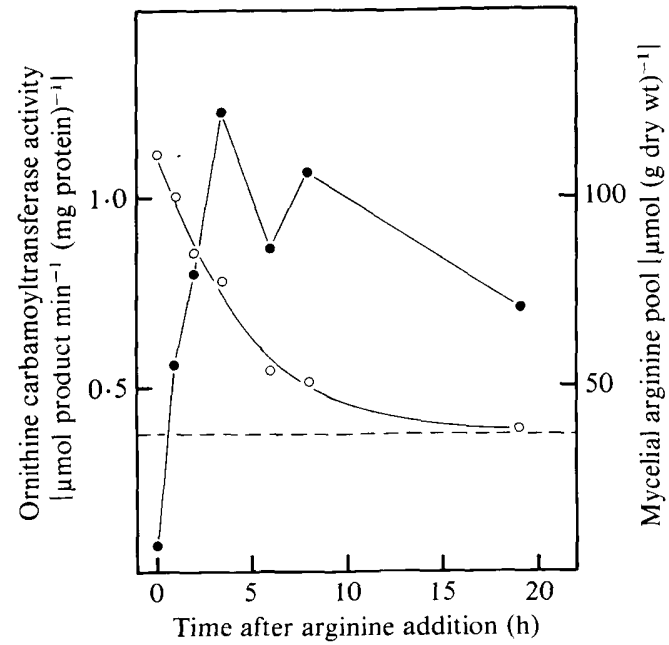

Fig. 3. Stability of ornithine carbamoyltransferase following repression by arginine in an arginine 'leaky' auxotroph. The decline in ornithine carbamoyltransferase activity $(O)$ and the increase in the mycelial arginine pool (O) are shown for the arginine 'leaky' auxotrophic strain arg-1r3 arg-10rl following the addition of arginine to the medium (to a concentration of $1.2 \mathrm{~mm}$ ). The dashed line indicates the activity observed in cultures grown on $1.2 \mathrm{~mm}$ arginine from the time of inoculation. Each point represents the mean of two replicate harvests; the values for individual replicates were within $5 \%$ of the mean value shown. The curve for the decline in enzyme activity represents the decline expected for a stable enzyme, based on the observed doubling time of $2.8 \mathrm{~h}$. |For a stable enzyme $a_{\mathrm{t}}=\left(a_{0}-a_{\mathrm{r}}\right) \mathrm{e}^{-k t}+a_{\mathrm{r}}$, where $k$ is the exponential growth constant, $a_{\mathrm{t}}$ is the activity per unit protein at time $t, a_{0}$ is the activity at $t_{0}$ when arginine is added and $a_{\mathrm{r}}$ is the 'fully repressed' activity.] 


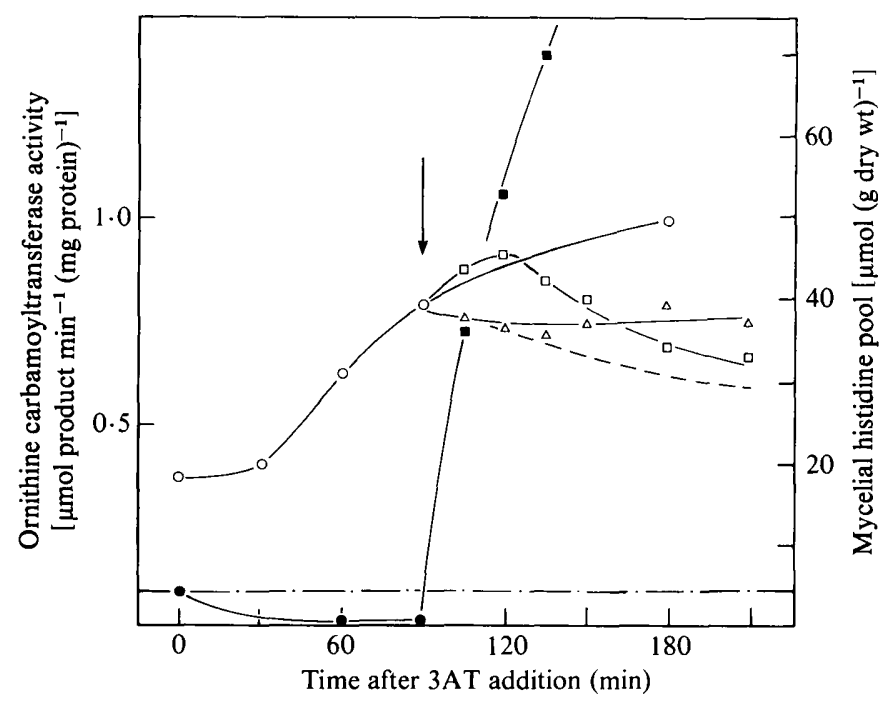

Fig. 4. Interrupted derepression of ornithine carbamoyltransferase. Ornithine carbamoyltransferase activity (open symbols) and the mycelial histidine pool (solid symbols) are shown for wild-type cultures to which 3 AT was added (final concentration $2.5 \mathrm{mM}$ ) after $17 \mathrm{~h}$ growth on minimal medium. Cultures either received no further addition $(O, O)$ or received histidine $(6 \mathrm{mM})(\square, \square)$ or histidine $(6 \mathrm{mM})$ plus cycloheximide $\left(12.5 \mu \mathrm{g} \mathrm{ml}^{-1}\right)(\triangle)$ at the time indicated by the arrow. The dashed line shows the decline in enzyme activity expected if the repressed rate of enzyme synthesis became restored immediately following the addition of histidine (in the absence of cycloheximide) (see text). The histidine level in minimal-grown cultures is also indicated $(-\cdot-\cdot)$. Each point represents the mean of two replicate harvests; the values for the two replicates typically differed from the mean value by less than $2.5 \%$, and never by more than $5 \%$.

including OCTase, during growth on minimal medium. The decline in OCTase activity (Fig. 3 ) following addition of arginine is accounted for simply by dilution of existing enzyme due to growth, and it may therefore be concluded that degradation of the enzyme is negligible under these conditions. Similar types of experiments were used to confirm that OCTase is also stable following the addition of arginine to starved $\arg -5$ cultures, following the addition of histidine to 3AT-treated wild-type cultures and following the inhibition of protein synthesis by cycloheximide.

\section{'Interrupted' derepression of ornithine carbamoyltransferase}

No 'lag' in the onset of repression that might be ascribed to the decay of derepressed OCTase synthetic capacity (i.e. OCTase-specific mRNA) was detected in the experiments described in the preceding section. However, because of the extended sampling periods employed this merely implies that, if control is transcriptional, the half-life of OCTase synthetic capacity is a matter of minutes rather than hours. In an experiment of shorter duration (Fig. 4), wild-type cultures were grown for $90 \mathrm{~min}$ in the presence of $3 \mathrm{AT}$ alone, by which time OCTase activity had risen to twice the level found during growth on minimal medium, before receiving either histidine alone or histidine and cycloheximide. The addition of cycloheximide, which is a potent inhibitor of translation at the concentration used here (Flint, 1977), prevented any further increase in enzyme activity and also prevented any further growth. Because OCTase is stable (see Fig. 3) its activity remained substantially constant in the presence of cycloheximide. The absence of any detectable lag in the effect of cycloheximide on OCTase activity in this experiment confirms that the increase in this enzyme caused by $3 \mathrm{AT}$ is due to de novo enzyme synthesis rather than the activation of an enzyme precursor. Although 
OCTase is a mitochondrial enzyme (Bernhardt \& Davis, 1972) this experiment does not suggest the existence of any inactive cytoplasmic precursor. In cultures that received histidine, but no cycloheximide, OCTase activity continued to increase for about $30 \mathrm{~min}$ before declining, while growth was found to be greater than in cultures grown in the presence of $3 \mathrm{AT}$ only (see Fig. 1). Had the addition of histidine resulted in an immediate return to the repressed rate of OCTase formation typical of minimal-grown cultures, an immediate decline in OCTase activity would have been observed. The dashed line in Fig. 4 shows the decline predicted on this basis, taking into account the growth rate of the culture subsequent to histidine addition. Two explanations for this lag in histidine action were considered. First, histidine may enter the mycelium slowly (despite the high concentration used $-6 \mathrm{~mm}$ ) and it may therefore take an appreciable time to attain mycelial histidine concentrations high enough to cause repression. Secondly, repression may be immediate and the lag due to the decay of derepressed OCTase synthetic capacity. The mycelial histidine content was found to respond rapidly to the presence of histidine in the medium (Fig. 4), reaching a level 10 times higher than that found in minimal-grown cultures $15 \mathrm{~min}$ after histidine addition. Since repressive conditions are therefore likely to have been restored within a few minutes of histidine addition, the explanation is favoured that the lag in histidine action represents the decay of OCTase synthetic capacity, present at high levels in the derepressed cultures. This would be consistent with control of OCTase formation exerted at the level of transcription or at the level of RNA processing or transport. A reliable estimate for the half-life of OCTase synthetic capacity cannot be obtained from these data. However, since the rate of increase in activity fell from about 5 milliunits $\mathrm{min}^{-1}$ to zero within $30 \mathrm{~min}$ of histidine addition (during which time the increase in total protein was less than $10 \%$ ) the half-life must clearly be considerably less than $30 \mathrm{~min}$ (see Fig. 4). Similar experiments performed with Saccharomyces cerevisiae have yielded estimates of 5 to $7 \mathrm{~min}$ for the half-life of OCTase synthetic capacity in that organism (Messenguy \& Cooper, 1977).

\section{DISCUSSION}

'Cross-pathway' regulation (i.e. the regulation of an enzyme by a metabolite belonging to a different pathway) has previously been observed for a number of enzymes involved in tryptophan, histidine and arginine synthesis in Neurospora crassa, under conditions of histidine or tryptophan limitation (Carsiotis et al., 1970; Carsiotis \& Jones, 1974; Carsiotis et al., 1974; Spurgeon \& Matchett, 1977). It has been taken to imply the existence of a 'general' control system governing enzymes in many different amino acid pathways and enabling them to respond to the levels of various amino acid end-products.

In the present study the six amino acid synthetic enzymes examined included four concerned with arginine synthesis, one with histidine synthesis and one with lysine synthesis. These enzymes were all found to display cross-pathway regulation, responding to reductions in histidine and in arginine. If a single general system of control governed the derepression of these enzymes in response to either amino acid, the relationship between the activities of any two enzymes (belonging to the same or different pathways) should be the same regardless of which amino acid is responsible for derepression. The evidence from this study (Fig. 2, Table 4) suggests that this is the case. Thus the response of the four arginine enzymes to conditions of arginine limitation can be accounted for solely through the action of the general control system. In yeast all eight enzymes involved in arginine synthesis are known to be subject to general control, but six of them (including ornithine carbamoyltransferase and acetylornithine aminotransferase) show an additional 'pathway-specific' form of control, for which arginine is the signal (Delforge et al., 1975; Messenguy, 1979). The latter system is responsible for the repression observed for most of the arginine enzymes in yeast when minimal-grown cultures are supplemented with arginine. The four enzymes examined in $N$. crassa in the present study do not appear to display such pathway-specific control, and are 
not repressed in wild-type cultures by exogenously provided arginine. Indeed, only one arginine synthetic enzyme (carbamoyl-phosphate synthetase) responds to exogenously provided arginine in $N$. crassa (Cybis \& Davis, 1975). This enzyme was not included in the present study, but has previously been shown to be subject to cross-pathway regulation (Carsiotis et al., 1974; Spurgeon \& Matchett, 1977). 'Dual' control by general and pathway-specific systems therefore remains a possibility for this enzyme.

The spectrum of amino acids capable of causing derepression of ornithine carbamoyltransferase has been studied in Aspergillus nidulans (Piotrowska, 1980) where it was found that starvation of auxotrophic strains for histidine, arginine, proline or tryptophan led to derepression whereas starvation for lysine, leucine or phenylalanine did not. A broadly similar pattern of control is found in $N$. crassa (unpublished results). Any proposed mechanism for general control must take account of the fact that many, but not all, amino acids appear to be capable of eliciting derepression.

Another important feature of cross-pathway regulation in $N$. crassa is that derepression resulting from the limitation of one amino acid is not reversed by increases in other amino acids (Carsiotis et al., 1970). In the present study the histidine-mediated derepression of arginine enzymes occurred despite being accompanied by increases in many other amino acid pools, and was not reduced by the presence of arginine in the medium. This is explained by the fact that enzyme levels are unresponsive to variation in any amino acid when this variation occurs at levels above those found in the minimal-grown wild-type. Thus supplementation of wild-type cultures with casein hydrolysate failed to produce any major repressive effect for the enzymes examined here. A detailed study of the relationship between mycelial arginine and the last three enzymes of arginine synthesis in arginine bradytrophic strains (Barthelmess et al., 1974) has shown that enzyme levels are insensitive to arginine at concentrations ranging from sixfold greater to fourfold less than those found in the minimal-grown wild-type. Further reductions, however, caused a more than proportionate increase in enzyme. This sharp response may be a significant property of the mechanism involved in general control. If so, similar responses should be found at low levels of the other amino acids capable of eliciting derepression.

Finally, the more detailed investigations reported here into the regulation of ornithine carbamoyltransferase lead to several conclusions concerning the nature of general control. The stability of the enzyme under a variety of conditions (see Fig. 3) effectively rules out control at the level of enzyme degradation. The requirement of de novo synthesis for enzyme depression is suggested by the effect of cycloheximide when added to cultures undergoing derepression, while the consequences of reversing histidine-mediated derepression through histidine addition are consistent with control at a stage prior to translation (see Fig. 4). More decisive support for this latter conclusion would come if it were possible to demonstrate that enzyme synthetic capacity could accumulate in the presence of a translational inhibitor, as has been shown for some inducible enzymes (Subramanian \& Sorger, 1972; Cybis \& Weglenski, 1972). However, such an approach cannot be used to study derepression in response to amino acid limitation because the inhibition of protein synthesis prevents amino acids becoming limited. Thus, in the immediate absence of specific inhibitors of transcription, temperature-sensitive blocks in transcription, or means of estimating specific mRNA concentrations, experiments of the type reported here provide the best available approach.

We are indebted to Dr H. Kacser for valuable discussion, for amino acid analysis and for providing two of the strains. This work is supported by Science Research Council Grant No. GR/A 72544.

\section{REFERENCES}

Barthelmess, I. B., Curtis, C. F. \& Kacser, H. (1974). Control of the flux to arginine in Neurospora crassa: de-repression of the last three enzymes of the arginine pathway. Journal of Molecular Biology 87 , 303-316.

BernhardT, S. A. \& Davis, R. H. (1972). Carbamoyl 
phosphate compartmentation in Neurospora: histochemical localization of aspartate and ornithine transcarbamoylases. Proceedings of the National Academy of Sciences of the United States of America 69, 1868-1872.

Broquist, H. P. (1971). Saccharopine dehydrogenase. Methods in Enzymology 17B, 124-129.

Carsiotis, M. \& Jones, R. F. (1974). Cross pathway regulation: tryptophan mediated control of histidine and arginine biosynthetic enzymes in Neurospora crassa. Journal of Bacteriology 119, 889-892.

Carsiotis, M., Jones, R. F., Lacy, A., Cleary, T. J. \& Fankhauser, D. B. (1970). Histidine mediated control of tryptophan biosynthetic enzymes in Neurospora crassa. Journal of Bacteriology 104, 98-106.

Carsiotis, M., Jones, R. F. \& Wesseling, A. C. (1974). Cross pathway regulation: histidine mediated control of histidine, tryptophan and arginine biosynthetic enzymes in Neurospora crassa. Journal of Bacteriology 119, 893-898.

Cybis, J. \& Davis, R. H. (1975). Organisation and control in the arginine biosynthetic pathway of Neurospora. Journal of Bacteriology 123, 196-202.

CyBis, J. \& Weglenski, P. (1972). Arginase induction in Aspergillus nidulans. The appearance and decay of the coding capacity of messenger. European Journal of Biochemistry 30, 262-268.

Davis, R. H. (1962). A mutant form of ornithine transcarbamylase found in a strain of Neurospora carrying a pyrimidine-proline suppressor gene. Archives of Biochemistry and Biophysics 97, 185191.

Davis, R. H., Weiss, R. L. \& Bowman, B. J. (1978). Intracellular metabolite distribution as a factor in regulation in Neurospora. In Microenvironments and Metabolic Compartmentation, pp. 197-210. Edited by P. A. Srere \& E. W. Estabrook. New York \& London: Academic Press.

Delforge, J., Messenguy, F. \& Wiame, J.-M. (1975). The regulation of arginine biosynthesis in Saccharomyces cerevisiae. The specificity of $\arg R^{-}$mutations and the general control of amino acid biosynthesis. European Journal of Biochemistry 57, 231-239.

DENES, G. (1970). Ornithine acetyltransferase (Chlamydomonas reinhardti). Methods in Enzymology 17A, 273-277.

FLINT, H. J. (1977). Compartmentation in the arginine pathway of Neurospora crassa. Ph.D. thesis, University of Edinburgh.
Flint, H. J., Porteous, D. J. \& Kacser, H. (1980). Control of flux in the arginine pathway of Neurospora crassa: the flux from citrulline to arginine. Biochemical Journal 190, 1-15.

Marsh, W. H., Fingerhut, B. \& Miller, H. (1965). Automated and manual direct methods for the determination of blood urea. Clinical Chemistry 11 , 624-627.

MARTIN, R. G. (1971). Histidinol dehydrogenase. Methods in Enzymology 17B, 36-37.

Messenguy, F. (1979). Concerted repression of the synthesis of the arginine biosynthetic enzymes by amino acids: a comparison between the regulatory mechanism controlling amino acid biosynthesis in bacteria and yeast. Molecular and General Genetics 169, 85-95.

Messenguy, F. \& Cooper, T. G. (1977). Evidence that specific and 'general' control of ornithine carbamoyltransferase production occurs at the level of transcription in Saccharomyces cerevisiae. Journal of Bacteriology 136, 1253-1261.

Piotrowska, M. (1980). Cross-pathway regulation of ornithine carbamoyltransferase synthesis in Aspergillus nidulans. Journal of General Microbiology 116, 335-339.

SChürCH, A., Miozzari, J. \& HütTER, R. (1974). Regulation of tryptophan biosynthesis in Saccharomyces cerevisiae: mode of action of 5 methyl tryptophan and 5-methyl tryptophan sensitive mutants. Journal of Bacteriology 117, 11311140 .

ScotT, W. A. (1975). Glucose-6-phosphate dehydrogenase from Neurospora crassa. Methods in Enzymology 41, 177-182.

Spurgeon, S. L. \& Matchett, W. H. (1977). Inhibition of aminoacyl-transfer ribonucleic acid synthetases and the regulation of amino acid biosynthetic enzymes in Neurospora crassa. Journal of Bacteriology 129, 1303-1312.

Subramanian, K. N. \& Sorger, G. J. (1972). Regulation of nitrate reductase in Neurospora crassa: regulation of transcription and translation. Journal of Bacteriology 110, 547-553.

Van Pilsum, J. F., Martin, R. P., Kito, E. \& Hess, J. (1956). Determination of creatine, creatinine, arginine, guanidoacetic acid, guanidine and methylguanidine in biological fluids. Journal of Biological Chemistry 222, 225-236.

Vogel, H. J. \& Jones, E. E. (1970). Acetyl ornithine $\delta$-aminotransferase (Escherichia coli). Methods in Enzymology 17A, 260-264. 